\title{
Target classification system based on the characterization of targets by subspaces
}

\author{
Jonathan Pisane ${ }^{*, \dagger}$, Marc Lesturgie ${ }^{\dagger}$, Jacques Verly* \\ *: Dept. of Electrical Engineering \& Computer Science, University of Liège, Grande Traverse 10, B-4000 Liège, Belgium \\ Phone: +32 4366 2642, e-mail: jpisane@ulg.ac.be \\ ${ }^{\dagger}$ : SONDRA, SUPELEC, Rue Joliot-Curie 3, 91192 Gif-sur-Yvette, France
}

\begin{abstract}
This paper presents a novel classification technique based on targets bistatic backscattering coefficients acquired at low frequencies. Each target class is characterized by a subspace. Air targets are then classified based on geometrical classification criteria. An average recognition rate of $90 \%$ is achieved.
\end{abstract}

\section{INTRODUCTION}

This paper presents a novel target classification method based on the bistatic backscattering coefficients of targets acquired at low frequencies, in the context of targets (passively) illuminated by transmitters of opportunity. The classification system is based on the characterization of a target by a particular subspace. The use of low frequencies offers several advantages. First, in the present context, low-frequency radio-communication signals are present everywhere in our environment, such as FM radio, digital television, and mobile phone communication networks. These signal sources are considered as transmitters of opportunity in the context of passive radar. Second, more generally, operating at low frequencies allows to defeat the possible stealthiness of targets.

In contrast to other approaches described in the literature, the goal is not to base the classification system upon the reconstructed images of the targets. Instead, it relies directly on their backscattering coefficients.

Section II shows a realistic configuration of the problem to be addressed. Section III details the algorithm that has been designed to classify the targets. Section V describes the experiments performed on the database, as well as their results. A short conclusion ends the report and emphasizes the future work that should be performed.

\section{Configuration}

A realistic configuration of the problem addressed is shown in Fig. 1. It is assumed that a flying target has been detected and that its location is known. Figure 1 shows three transmitters $\left(T x_{i}\right)$ illuminating the target, and one receiver $(R x 1)$ collecting the signals reflected by the target. The desired backscattering coefficients are obtained from the signal collected at the receiver. It is assumed that the frequency band $f$ and the polarization $P(\mathrm{H}$ or $\mathrm{V})$ of the transmitters, as well as the angular sector $\theta$ that the target is detected in, are known. A "model" must thus be built for each triplet $\{f, P, \theta\}$.

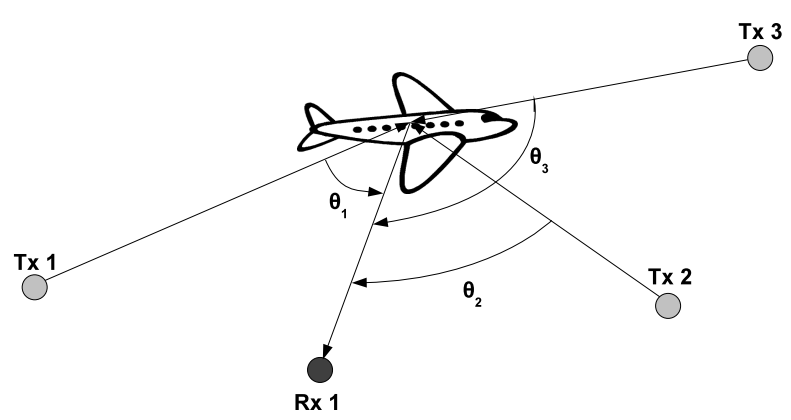

Fig. 1. General system configuration for three transmitters and one receiver. The target is an air vehicle.

\section{ClaSSIFICATION ALGORITHM}

The classification system is divided in two parts. First, the classification model, based on the characterization of the targets by subspaces, must be built. Then, the classification of the targets can be performed, according to the model built. Note that the database is divided in two parts. The learning set (LS) is used to build the model, and the test set (TS) contains targets to be classified.

\section{A. Construction of model}

The subspace model consists of $N_{\text {Class }}$ subspaces per triplet $\{f, P, \theta\}$, where $N_{\text {Class }}$ is the number of the different target classes. The following operations are performed to build the model (Fig. 2). First, the LS vectors are separated according to their corresponding class, forming 
$N_{\text {Class }}$ sub-learning sets. The division of the data into classes is discussed in Section V. Then, among each sublearning set, the data vectors are divided according to the triplet $\{f, P, \theta\}$ each data vector belongs to. The triplet $\{f, P, \theta\}$ is known for each vector, by construction of the system. Thus, the original LS has been divided into $N_{\text {Class }} * N_{\text {Triplet }}$ subsets. One subspace is built for each one of these subsets.

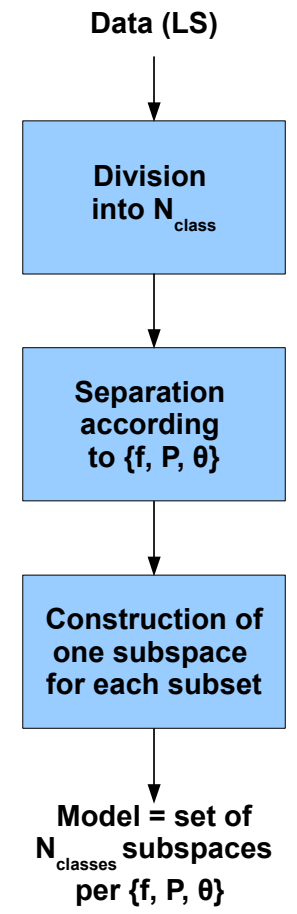

Fig. 2. Construction of the subspace model. The LS vectors are separated according to their class and triplet $\{f, P, \theta\}$. The subspaces are then computed by performing an SVD on each subset.

Each subspace is computed by performing a singular value decomposition (SVD) of the training data $H$ belonging to each subset [1],

$$
H=U \Lambda V^{\dagger}
$$

where $\Lambda$ is a diagonal matrix of the singular values, $U$ the matrix containing the corresponding singular vectors related to $H H^{\dagger}$, and $V$ the matrix containing the singular vectors related to $H^{\dagger} H$, where $\dagger$ denotes the conjugate transpose operator.

The orthogonal projector $P_{z}$ is then built, for each subspace (1 subspace per class), and for each triplet.

$$
P_{z}=U U^{\dagger}
$$

where $\dagger$ denotes the complex conjugate. For computational reasons, each subspace is of size 4 . The vectors corresponding to the 4 most significant singular values are kept.

\section{B. Classification according to model}

Once the model is built, the vectors of the test set (TS) can then be classified. The procedure is shown in Fig. 3, for one vector $z$ of TS. First, the triplet $\{f, P, \theta\}$ vector $z$ belongs to has to be determined. The triplet each vector belongs to is known, this by construction of the system. Then, vector $z$ is classified according to the three different classification tests that are described in Section IV. Finally, a combination of the three tests is performed to give an overall class to $z$ (Section IV-D).

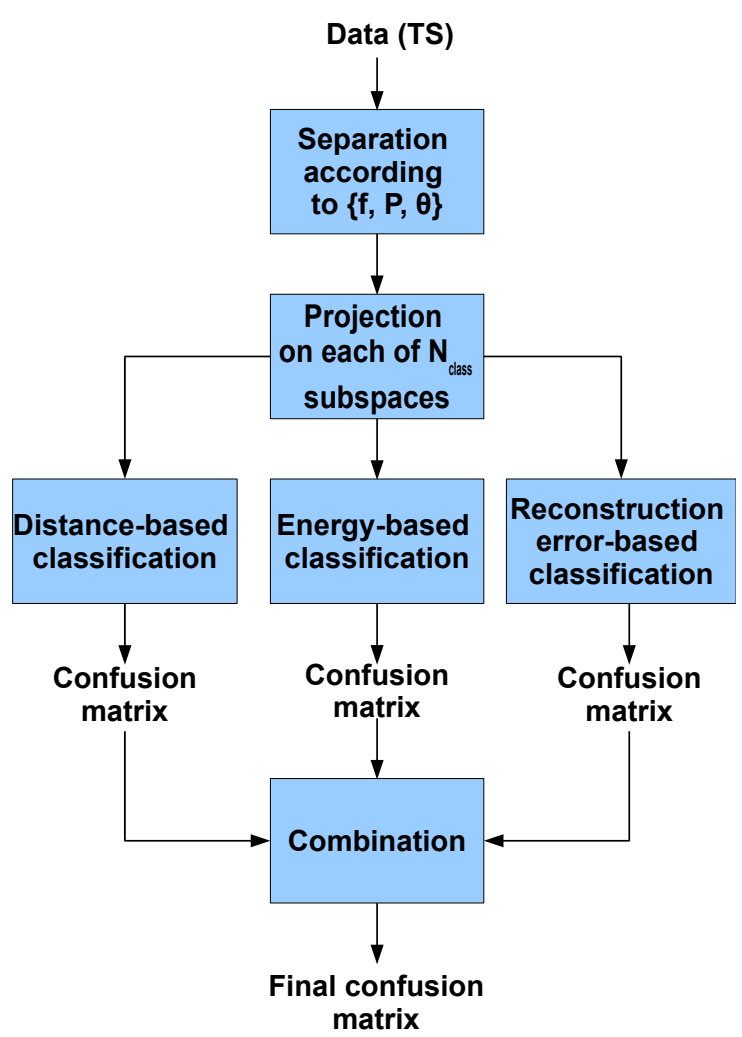

Fig. 3. Classification of one vector of the test set. The triplet $\{f, P, \theta\}$ the vector belongs to is determined. Then, the projection of the vector into each one of the $N_{\text {Class }}$ subspaces is computed. The vector is classified for each one of the three classification tests used.

\section{ClassificAtion tests}

Three classification tests have been implemented. They are all based on the projections of a vector $z$ to be classified into each one of the $N_{\text {Class }}$ subspaces of one triplet $\{f, P, \theta\}$.

\section{A. Test based on energy}

The energy-based classification test works as follows. To classify a test vector $z$, one first computes the energy 
of its projection $E_{z}$ into each of the $N_{\text {Class }}$ subspaces according to,

$$
E_{z}=z^{H} P_{z} z
$$

The test vector is then assigned the class for which the percentage of energy of its projection is the highest, where the percentage of energy of a vector $z$ in each subspace is computed as

$$
\operatorname{PercE} E_{z}=E_{z} / z^{H} z
$$

\section{B. Test based on distance [2]}

The distance-based classification test works as follows. The squared orthogonal projection distance $d(z, L)$ between a subspace $L$ and a vector $z$ to classify is computed, according to

$$
d(z, L)=\sum_{i=1}^{i=m}\left(z^{T} l_{i}\right)^{2},
$$

where $l_{i}$ are the vectors of the subspace $L$, and $m$ the dimension of the vector $z$.

The classification criterion is the following. If $d\left(z, L^{(i)}\right)>d\left(z, L^{(j)}\right), i \neq j$, then $z$ belongs to class $i$.

\section{Test based on reconstruction error [2]}

The reconstruction error-based classification test works as follows. From the projection of $z$ into a subspace, we can reconstruct the original vector $\hat{z}$ :

$$
\hat{z}=\sum_{i=1}^{i=m}\left(z^{T} l_{i}\right) l_{i} .
$$

From this reconstruction, a residual error $\tilde{z}$ can be computed:

$$
\tilde{z}=z-\hat{z} \text {. }
$$

The classification criterion is the following. Vector $z$ will be given the class for which its residual error $\tilde{z}$ is the lowest, among the $N_{\text {Class }}$ residual errors computed for a particular triplet $\{f, P, \theta\}$.

\section{Combinations of the classification tests}

Classification tests have been described for one transmitter and one receiver. The idea is to combine the classification resulting from different transmitters. Two physical parameters were taken into account. First, the classification results were combined among one polarization (either $\mathrm{HH}$, $\mathrm{HV}, \mathrm{VH}$, or VV), for different frequency bands. That simulates the fact that different transmitters operating on different frequency bands but on the same polar could be used in the classification system. Second, the combination resulted on the polarization of both the transmitter and the receiver. That means that all the transmitters considered emit their signal in a different polarization, but the frequency band could be the same. The angular sector could in both cases be the same or different from one transmitter to the other.

\section{CLASSIFICATION EXPERIMENTS}

\section{A. Description of the database}

The database of interest consists of the bistatic backscattering coefficients of four types of targets. These target types are F16, F117, Learjet, and Beechcraft airplanes. The backscattering coefficients were measured in an anechoic chamber, in bistatic mode, at four different frequency bands $f_{i}$, four polarizations $P_{j}(\mathrm{HH}, \mathrm{HV}, \mathrm{VH}$, and $\mathrm{VV}$, where the first letter indicates the polarization of the transmitter, and the second letter the polarization of the receiver), and six different angular sectors $\theta_{k}$. This gives a total of $6 * 4 * 4=96$ triplets $\left\{f_{i}, P_{j}, \theta_{k}\right\}$. For each triplet $\left\{f_{i}, P_{j}, \theta_{k}\right\}$, measurements were taken for the targets at different elevation and orientation angles. One vector of backscattering coefficients for one orientation angle and one elevation angle constitutes one object, per triplet $\left\{f_{i}, P_{j}, \theta_{k}\right\}$ and per target. Thus, the vectors of each subspace are constituted by the vectors containing the backscattering coefficients of a particular target class, for a given triplet $\left\{f_{i}, P_{j}, \theta_{k}\right\}$, each vector corresponding to one elevation angle and one orientation angle.

\section{B. Classification according to energy}

Table I presents the confusion matrices for the $\mathrm{HH}$ polarization. Results are similar for the $\mathrm{HV}, \mathrm{VH}$, and VV polarizations. It indicates that the F16 have always a high classification rate (about 80\%), while the classification rate is comprised between $60 \%$ and $80 \%$ for the other targets. It also shows that the division of the target into those four classes is homogeneous. No target is better classified in a class (other than its own) than in another class. The test can thus be considered as valid.

\section{Classification according to distance}

Table II presents the confusion matrices for the $\mathrm{HH}$ polarization. Results are similar for the $\mathrm{HV}, \mathrm{VH}$, and $\mathrm{VV}$ polarizations. The same conclusions can be drawn as for the energy-based test. 


\section{Classification according to reconstruction error}

Tables III presents the confusion matrices for the $\mathrm{HH}$ polarization. Results are similar for the $\mathrm{HV}, \mathrm{VH}$, and VV polarizations. All classification rates are quite low. This indicates that the test might be inaccurate. No other conclusion can therefore be drawn from this test.

\section{E. Combination of the three classification tests}

Table IV shows the confusion matrix for a combination of the three classification tests, triplet per triplet, and object per object, for the $\mathrm{HH}$ polarization. Here again, results are similar for the $\mathrm{HV}, \mathrm{VH}$, and $\mathrm{VV}$ polarizations. The result is obtained by performing a majority vote among the three different tests.

1) Combining different transmitters at different frequency bands: Three transmitters have been combined, each one emitting at a different frequency band, and at the same polarization. Since four frequency bands and four polarizations are considered, there are 16 confusion matrices. It appears that almost all the classification rates are comprised between $70 \%$ and $95 \%$ (Table $\mathrm{V}$ for example).It also appears that the $\mathrm{V}$ polarization at transmitter is better than the $\mathrm{H}$ polarization. Moreover, many objects are either correctly classified, or are undetermined. Undetermined means that there was no majority for one class among the three classes assigned to an object, one class per frequency band. The undetermined cases can be explained by the fact that the reconstruction-error test is not very relevant. Therefore, we will focus on the undetermined cases in our next work by trying other classification tests.

2) Combining different transmitters at different polarizations: Three couples transmitters-receiver have been combined, each one emitting or receiving at a different polarization. This means that for each one of the three objects considered, its backscattering coefficients can have been registered either at $\mathrm{HH}, \mathrm{HV}, \mathrm{VH}$, or $\mathrm{VV}$. This gives us four confusion matrices (Table VI for example).Since there are 27 objects per triplet, and 24 triplets per polarization, each polarization contains $27 * 24=648$ classified objects Each object has been classified taking into account the three individual classification tests. When combining three polarizations out of the four, it gives us $648^{3}=272097792$ cases. The confusions matrices show that the correct classification rates all lie between $70 \%$ and $90 \%$. The number of undetermined objects is also very high, between $10 \%$ and $25 \%$ of the objects. This might also be due to the reconstruction-error test.

\section{CONCLUSION AND FUTURE WORK}

We have presented a novel classification system that classifies targets based on their bistatic backscattering co- efficients. The classification system characterizes a target class by a subspace, and then uses three different tests to classify any object. The classification tests are all a different measure of the representation of an object in a subspace, either an energy measure, a distance measure, or a reconstruction measure. The combination of the tests have also been performed. The classification system has been tested on a database of bistatic backscattering coefficients acquired in an anechoic chamber. The correct classification rates are comprised between $70 \%$ and $95 \%$. Simulations of three transmitters at different frequency bands and different polarizations have also been performed. The correct classification rates are comprised between $70 \%$ and $90 \%$.

A large number of objects could not be attributed a class, and were then left undetermined. This is due to the poor performance of the reconstruction error-based test. Therefore, more efficient tests will be studied. Moreover, the classification system will be tested on more realistic databases.

\section{REFERENCES}

[1] L. Scharf and C. Demeure, Statistical Signal Processing: Detection, Estimation, and Time Series Analysis. Addison-Wesley Reading, MA, 1991.

[2] H. Bischof, A. Leonardis, and F. Pezzei, "A robust subspace classifier," in International Conference on Pattern Recognition, vol. 14, pp. 114-116, Citeseer, 1998. 


\begin{tabular}{|l|l|l|l|l|}
\hline $\begin{array}{l}\text { True vs. } \\
\text { recog- } \\
\text { nized }\end{array}$ & Beech & F117 & F16 & Learjet \\
\hline Beech & 66.98 & 15.12 & 8.18 & 9.72 \\
\hline F117 & 5.56 & 64.51 & 11.73 & 9.1 \\
\hline F16 & 2.93 & 5.86 & 84.41 & 4.17 \\
\hline Learjet & 8.18 & 8.49 & 14.66 & 62.5 \\
\hline
\end{tabular}

TABLE I

THE CONFUSION MATRIX SHOWS THE CORRECT CLASSIFICATION RATE FOR EACH CLASS OF TARGET (IN \%) FOR THE ENERGY-BASED TEST, AND CONSIDERING ONLY THE HH POLARIZATION. IT SHOWS

THAT THE CLASSIFICATION RATES ARE QUITE HOMOGENEOUS.

\begin{tabular}{|l|l|l|l|l|}
\hline $\begin{array}{l}\text { True vs. } \\
\text { recog- } \\
\text { nized }\end{array}$ & Beech & F117 & F16 & Learjet \\
\hline Beech & 64.66 & 17.59 & 7.41 & 10.34 \\
\hline F117 & 6.94 & 69.75 & 14.04 & 9.26 \\
\hline F16 & 3.55 & 9.72 & 82.25 & 4.48 \\
\hline Learjet & 10.19 & 9.41 & 18.52 & 61.88 \\
\hline
\end{tabular}

TABLE II

THE CONFUSION MATRIX SHOWS THE CORRECT CLASSIFICATION RATE FOR EACH CLASS OF TARGET (IN \%) FOR THE DISTANCE-BASED TEST, AND CONSIDERING ONLY THE HH POLARIZATION. IT SHOWS THAT THE CLASSIFICATION RATES ARE QUITE HOMOGENEOUS.

\begin{tabular}{|l|l|l|l|l|}
\hline $\begin{array}{l}\text { True vs. } \\
\text { recog- } \\
\text { nized }\end{array}$ & Beech & F117 & F16 & Learjet \\
\hline Beech & 53.55 & 16.82 & 11.57 & 18.06 \\
\hline F117 & 12.96 & 52.78 & 16.82 & 17.44 \\
\hline F16 & 12.81 & 15.74 & 57.56 & 13.89 \\
\hline Learjet & 9.41 & 17.44 & 18.52 & 54.63 \\
\hline
\end{tabular}

TABLE III

THE CONFUSION MATRIX SHOWS THE CORRECT CLASSIFICATION RATE FOR EACH CLASS OF TARGET (IN \%) FOR THE

RECONSTRUCTION ERROR-BASED TEST, AND CONSIDERING ONLY THE HH POLARIZATION. IT SHOWS THAT THE CLASSIFICATION RATES ARE QUITE HOMOGENEOUS AND LOW.

\begin{tabular}{|l|l|l|l|l|l|}
\hline $\begin{array}{l}\text { True } \\
\text { vs. } \\
\text { recog- } \\
\text { nized }\end{array}$ & Beech & F117 & F16 & Learjet & Und \\
\hline Beech & 66.36 & 15.12 & 5.71 & 9.57 & 3.24 \\
\hline F117 & 5.56 & 63.73 & 12.96 & 8.64 & 9.1 \\
\hline F16 & 3.24 & 7.1 & 82.1 & 4.32 & 3.24 \\
\hline Learjet & 7.1 & 8.18 & 14.51 & 62.96 & 7.25 \\
\hline
\end{tabular}

TABLE IV

THE CONFUSION MATRIX SHOWS THE CORRECT CLASSIFICATION RATE FOR EACH CLASS OF TARGET (IN \%) FOR THE COMBINATION OF THE THREE CLASSIFICATION TESTS, AND CONSIDERING ONLY THE HH POLARIZATION. IT SHOWS THAT THE CLASSIFICATION RATES ARE QUITE HOMOGENEOUS AND LOW.

\begin{tabular}{|l|l|l|l|l|l|}
\hline $\begin{array}{l}\text { True } \\
\text { vs. } \\
\text { recog- } \\
\text { nized }\end{array}$ & Beech & F117 & F16 & Learjet & Und \\
\hline Beech & 78.1 & 3.48 & 0.32 & 3.27 & 14.82 \\
\hline F117 & 0.7 & 79.26 & 0.27 & 0.48 & 16.85 \\
\hline F16 & 0.21 & 0.92 & 94.87 & 0.05 & 3.95 \\
\hline Learjet & 2.43 & 1.79 & 2.94 & 71.92 & 20.92 \\
\hline
\end{tabular}

TABLE V

THE CONFUSION MATRIX SHOWS THE CORRECT CLASSIFICATION RATE FOR EACH CLASS OF TARGET (IN \%) FOR THE COMBINATION OF THREE TRANSMITTERS, EACH ONE EMITTING AT A DIFFERENT FREQUENCY BAND, FOR THE POLARIZATION HH. IT SHOWS THAT THE CLASSIFICATION RATE IS HIGH FOR ALL CLASSES, AND THAT A LARGE NUMBER OF OBJECTS ARE STILL UNDETERMINED.

\begin{tabular}{|l|l|l|l|l|l|}
\hline $\begin{array}{l}\text { True } \\
\text { vs. } \\
\text { recog- } \\
\text { nized }\end{array}$ & Beech & F117 & F16 & Leajet & Und \\
\hline Beech & 72.01 & 2.31 & 2.66 & 3.28 & 19.74 \\
\hline F117 & 2.64 & 70.03 & 3.54 & 0.98 & 22.55 \\
\hline F16 & 0.99 & 0.67 & 89.17 & 0.66 & 8.51 \\
\hline Learjet & 1.47 & 0.7 & 2.58 & 79.81 & 15.44 \\
\hline
\end{tabular}

TABLE VI

THE CONFUSION MATRIX SHOWS THE CORRECT CLASSIFICATION RATE FOR EACH CLASS OF TARGET (IN \%) FOR THE COMBINATION OF THREE TRANSMITTERS, EACH ONE EMITTING AT A DIFFERENT POLARIZATION HH, HV, OR VH. IT SHOWS THAT THE

CLASSIFICATION RATE IS HIGH FOR ALL CLASSES, AND THAT A LARGE NUMBER OF OBJECTS ARE STILL UNDETERMINED. 